\title{
Difference in Temperament and Character Inventory scores between depressed patients with bipolar II and unipolar major depressive disorders
}

\author{
Daimei Sasayama ${ }^{\mathrm{a}, \mathrm{b}}$, Hiroaki Hori ${ }^{\text {a }}$, Toshiya Teraishi ${ }^{\text {a }}$, Kotaro Hattori ${ }^{\text {a }}$, Miho Ota ${ }^{\text {a }}$, \\ Junko Matsuo $^{\text {a }}$, Yumiko Kawamoto a, Yukiko Kinoshita ${ }^{\text {a }}$, Miyako Hashikura ${ }^{\text {a }}$, \\ Norie Koga ${ }^{\text {a }}$, Nagahisa Okamoto ${ }^{\mathrm{c}}$, Kota Sakamoto $^{\mathrm{c}}$, Teruhiko Higuchi $^{\mathrm{d}}$, \\ Naoji Amano $^{\text {b }}$, Hiroshi Kunugi ${ }^{\text {a }}$
}

a Department of Mental Disorder Research, National Institute of Neuroscience, National Center of Neurology and Psychiatry, 4-1-1, Ogawahigashi, Kodaira, Tokyo, 187-8502, Japan

${ }^{\mathrm{b}}$ Department of Psychiatry, Shinshu University School of Medicine, 3-1-1 Asahi, Matsumoto, 390-8621, Japan

${ }^{\mathrm{c}}$ Department of Psychiatry, National Center Hospital, National Center of Neurology and Psychiatry, Kodaira, 187-8502, Japan

${ }^{\mathrm{d}}$ National Center of Neurology and Psychiatry, Kodaira, 187-8502, Japan

e JST, CREST, Japan

${ }^{\S}$ Correspondence to:

Daimei Sasayama

Department of Mental Disorder Research, National Institute of Neuroscience, National Center of Neurology and Psychiatry

4-1-1, Ogawahigashi, Kodaira, Tokyo, 187-8502, Japan

TEL: +81-42-341-2712, extension 5132

FAX: +81-42-346-1744

Email: sasayama@shinshu-u.ac.jp 


\section{Abstract}

Background: Although some core personality variables are known to be characteristic of unipolar or bipolar depression, few studies have compared the personality profile between these two disorders.

Methods: Temperament and Character Inventory (TCI) was employed to assess the personality of 36 depressed patients with bipolar II disorder (BPII), 90 patients with unipolar major depressive disorder (UP), and 306 healthy controls. The TCI was administered during the depressive episode in BPII and UP patients so that the results can be applied in a clinical setting.

Results: Significantly higher scores in harm avoidance (pb0.0001) and lower scores in selfdirectedness (pb0.0001) and cooperativeness (pb0.05) were observed in both BPII and UP patients compared to controls. Lower novelty seeking in UP patients compared to BPII patients and controls was observed in females (pb0.0001, pb0.01, respectively). A significant difference in self-transcendence score was observed between BPII and UP patients in females (pb0.0005), with higher scores in BPII $(\mathrm{p}=0.009)$ and lower scores in $\mathrm{UP}(\mathrm{p}=0.046)$ patients compared to controls. A logistic regression model predicted BPII 
in depressed females based on novelty seeking and self-transcendence scores with a sensitivity of $89 \%$ and a specificity of $73 \%$, but did not accurately predict BPII in males.

Limitations: Patients in our study were limited to those receiving outpatient treatments, and bipolar patients were limited to those with BPII.

Conclusions: Novelty seeking and self-transcendence scores of TCI might be useful in the differentiation of UP and BPII in female patients.

Keywords: Unipolar depression; Bipolar disorder; Personality; Differential diagnosis 


\section{Introduction}

Differentiating unipolar and bipolar depression is of great clinical importance since the treatment of the two disorders differs substantially. However, the differentiation may be difficult due to the similar depressive symptomatology in these two disorders. One study reported that $69 \%$ of bipolar disorder (BP) had been misdiagnosed, with the most frequent misdiagnosis being unipolar depression (Hirschfeld et al., 2003). Some clinical characteristics such as hypersomnia and psychotic features have been suggested to be more common in bipolar depression than in unipolar depression (Forty et al., 2008; Mitchell et al., 2001). However, there are no clear-cut clinical features that distinguish the two disorders, and clinicians must use all available information to predict the possibility of bipolarity in depressed patients.

A few studies have attempted to examine differences in personality between unipolar and bipolar depression. Two studies (Mendlowicz et al., 2005; Nowakowska et al., 2005) used the Temperament Evaluation of Memphis, Pisa, Paris, and San Diego-Autoquestionnaire (TEMPS-A) and found that cyclothymic subscale score was significantly elevated in BP patients. Another study (Lovdahl et al., 2010), which used 
Temperament and Character Inventory (TCI) (Cloninger et al., 1993) to compare bipolar II disorder (BPII) with recurrent brief depression (RBD; defined as intermittent depressive episodes fulfilling the diagnostic criteria for major depressive episodes except for duration, which is less than 14 days), failed to find definitive difference between the two disorders. Evans, et al. (Evans et al., 2005) used both TEMPS-A and TCI to find higher dysthymic, cyclothymic, irritable, anxious, and novelty seeking temperaments and lower self-directedness and cooperativeness characters in BP patients. A study using a battery including 17 conventional personality scales has reported sanguine in patients with bipolar I disorder, labile or cyclothymic in patients with BPII, and subanxious and subdepressive in patients with unipolar depression (Akiskal et al., 2006).

Although above studies report a number of findings showing different personality in UP and BP patients, few have assessed the personality of the two disorders during depressive state (Mendlowicz et al., 2005). Since the severity of depressive symptoms impact on how the subject describes their personality (Spittlehouse et al., 2010), it is important that the mood state at the time of assessment is 
taken into account. If the personality assessment is to be used as an aid for

differentiating unipolar and bipolar depression in the real world setting, studies are

required to compare personality measures during depressed mood.

In the present study, TCI was used to assess the personality difference between depressed BPII and UP patients. TCI is a 240 item true/false questionnaire measuring four dimensions of temperament (novelty seeking (NS), harm avoidance (HA), reward dependence (RD), and persistence (P)) and three dimensions of character (self-directedness (SD), cooperativeness (C), and self-transcendence (ST)), developed on the basis of a psychobiological model of personality. Although TCI has been frequently used in the studies of mood disorders (Akiskal et al., 2005; Celikel et al., 2009; de Winter et al., 2007; Engstrom et al., 2004; Farmer et al., 2003; Hansenne et al., 1999; Hirano et al., 2002; Kimura et al., 2000; Loftus et al., 2008; Marijnissen et al., 2002; Matsudaira and Kitamura, 2006; Naito et al., 2000; Richter et al., 2000; Smith et al., 2005), no studies to date have compared TCI score profiles of patients with unipolar and bipolar depression during their depressed states. We aimed to identify personality profiles specific to either unipolar or bipolar depression, which could aid in the 
differentiation of the two disorders.

\section{Methods}

\subsection{Subjects}

Subjects were 36 patients with BPII (18 men, 18 women; age \pm S.D. (standard deviation $)=36.3 \pm 11.1$ years), 90 patients with UP (45 men, 45 women; age \pm S.D. $=36.7 \pm 10.2$ years), and 306 healthy volunteers (153 men, 153 women; age \pm S.D. $=36.4 \pm 11.0$ years), matched for age distribution in each gender group, recruited from the outpatient clinic of the National Center of Neurology and Psychiatry Hospital, Tokyo, Japan or through advertisements in local free magazines, website announcement, notices posted in the hospital, flyers, and word of mouth. Consensus diagnoses by at least two research psychiatrists were made according to the DSM-IV criteria (American Psychiatric Association, 1994) for BPII or UP for enrollment in the study. Those recruited from the outpatient clinic (BPII: 24 patients (67\%); UP: 66 patients (73\%)) were also assessed with the Structured Clinical Interview for DSM-IV by a trained psychiatrist to confirm the diagnosis. BPII and UP patients with Hamilton Rating Scale 
for Depression (17-item version) score greater than 7 were enrolled in the study.

Healthy participants were interviewed using the Japanese version of the

Mini-International Neuropsychiatric Interview (Otsubo et al., 2005; Sheehan et al., 1998) by a research psychiatrist, and only those who demonstrated no history of psychiatric illness or contact to psychiatric services were enrolled as healthy controls. Participants were excluded from both the patient and control groups if they had a prior medical history of central nervous system disease or severe head injury, or if they met DSM-IV criteria for mental retardation, substance dependence, or substance abuse. All subjects were biologically unrelated Japanese who resided in the Western part of Tokyo. Written informed consent was obtained from all subjects prior to their inclusion in the study and the study was approved by the ethics committee of the National Center of Neurology and Psychiatry, Japan.

\subsection{Measures}

All participants were administered the TCI (Cloninger et al., 1993), a 240 item true/false self-report questionnaire measuring four dimensions of temperament and three 
dimensions of character. The Japanese version of the TCI (Kijima et al., 1996; Kijima et al., 2000) was used in the present study. Each subject was allowed to take as much time as needed to complete the questionnaire. Depressive symptoms were assessed by an experienced research psychiatrist using the Japanese version of the GRID Hamilton Rating Scale for Depression, 17-item version (HDRS) (Hamilton, 1967), which has been demonstrated to show excellent inter-rater reliability (Tabuse et al., 2007).

\subsection{Statistical analyses}

Gender differences concerning the temperament and the character dimensions have been reported previously (Gutierrez- Zotes et al., 2004; Hansenne et al., 2005; Pelissolo and Lepine, 2000), and thus is one of the major potential confounding factors in TCI studies. Therefore, the data for male and female subjects were analyzed separately to avoid gender-dependent influence.

Statistical differences of demographic data among groups were evaluated by the chi-squared test for categorical variables and one-way analysis of variance (ANOVA) for continuous variables. Student t-test was used for comparisons of clinical 
variables between BPII and UP patients. The scores of the dimensions of TCI were compared among the three diagnostic groups using Kruskal-Wallis test, and thereafter, pairwise comparisons between each group were done using Mann-Whitney tests. Bonferroni method was used to correct for multiple comparisons among the three diagnostic groups. However, since the scale scores of each TCI dimension are intercorrelated and thus are not completely independent measures, we did not apply Bonferroni method for the number of TCI dimensions. Correlations between TCI scores and HDRS scores were assessed using Spearman's rank correlation coefficients. A stepwise logistic regression analysis was used in patients with BPII and UP to determine the optimal model for the prediction of BPII in depressive patients. Prediction models were developed separately for males, females, and both genders combined. The stepwise analysis was conducted as a forward stepping procedure based on a likelihood ratio test, with pb0.05 for variable inclusion and $\mathrm{pN} 0.2$ for exclusion from the model. Variables used as potential predictor variables were age, gender, HDRS score, and scores of 7 dimensions of TCI. Nagelkerke's R2 was used to approximate the percent of variance explained by the model (Nagelkerke, 1991). The area under the receiver 
operating characteristic (ROC) curve (AUC) was also used to determine the predictive power of the logistic model. The predicted probability with the highest Youden index was selected as the optimal cut-off point. Statistical significance was set at two-tailed $\mathrm{pb} 0.05$. Analyses were performed using the SPSS version 11.0 (SPSS Japan, Tokyo).

\section{Results}

Table 1 shows demographic and clinical characteristics of the subjects. Age distribution did not differ across the three diagnostic groups. Although the average years of education were significantly higher in the male controls, there was no significant difference between BPII and UP patients. Patients with BPII and UP did not differ significantly in age at onset or in HDRS scores.

The mean scores for the 7 dimensions of TCI are presented in Table 2 . Three-group comparisons of BPII, UP patients, and controls revealed differences in all dimensions except for RD in both genders, $\mathrm{P}$ in females, and $\mathrm{ST}$ in males. Significantly higher HA and lower SD and C scores in BPII and UP patients compared with control subjects were observed in both males and females. Significantly higher P scores in BPII 
and UP patients were detected only in females. Lower NS in UP patients compared to BPII patients and healthy controls was observed only in females. ST scores in female subjects showed the opposite directions between BPII and UP patients, i.e. BPII patients scored higher and UP patients scored lower in ST compared to controls. The comparison of ST scores in female subjects between the UP patients and controls nearly reached the Bonferroni-corrected significance of pb0.017, and the comparisons of BPII patients with control subjects and with UP patients remained significant after Bonferroni correction.

The HDRS scores of UP patients were significantly correlated positively with $\mathrm{P}$ in female patients (rho $=0.327, \mathrm{pb} 0.05)$ and negatively with $\mathrm{SD}$ in male patients $(\mathrm{rho}=$ $-0.407, \mathrm{pb} 0.01)$. The HDRS in male BPII patient group was significantly correlated positively with $\mathrm{C}$ score $(\mathrm{rho}=0.497, \mathrm{pb} 0.05)$. No other significant correlations were found between TCI and HDRS scores.

Results of the stepwise logistic regression analysis are shown in Table 3. The Nagelkerke R2 values show that $8.7 \%, 40.3 \%$, and $24.1 \%$ of the variance are explained by the models for males, females, and both genders combined, respectively. The total 
AUC was significantly greater than 0.5 in models for females and for both genders combined but not for the model for males. These results indicate that the logistic regression model for female patients and for both genders combined appropriately fit the data while the model for male patients does not accurately predict BPII and UP. The following prediction model for female patients was derived:

Predicted Probability $=1 /\{1+\exp (-5.8143+0.129601 \times($ NS score $)+$ $0.20554 \times($ ST score $))\}$

At the optimal cut-off point of 0.782 determined by the Youden index, the sensitivity and the specificity of differentiating BPII from UP were 89\% and 73\%, respectively.

\section{Discussion}

The main findings of the present study could be summarized as follows. Higher HA, lower SD, and lower C scores were observed in both BPII and UP patients when compared with controls. In females, ST scores significantly differed between BPII and UP patients, with higher scores in BPII patients and lower scores in UP patients 
compared to controls. Patients with UP showed lower NS scores compared to controls in both genders and also to BP patients in females. BPII in depressed females could be predicted using the NS and ST scores.

Consistent with our results, several previous studies have shown higher scores on HA and lower scores on SD and C in patients with UP (Farmer et al., 2003; Hansenne et al., 1999) or in those with BP (Engstrom et al., 2004; Evans et al., 2005) compared to healthy controls. Higher NS (Evans et al., 2005; Nowakowska et al., 2005) and ST (Evans et al., 2005; Loftus et al., 2008; Nowakowska et al., 2005) have also been reported in BP patients compared to controls, though most studies comparing UP patients and controls have shown no significant difference in these two dimensions (Celikel et al., 2009; Evans et al., 2005; Farmer et al., 2003; Hansenne et al., 1999; Kimura et al., 2000; Marijnissen et al., 2002; Nowakowska et al., 2005; Smith et al., 2005). Although the comparison between BPII and UP patients showed similar trends in both genders (i.e., higher NS and ST in BPII), statistical significance between these two disorders was reached only in females. This suggests that, despite the similar tendency in both genders, the differences between diagnostic groups in TCI profiles are more 
evident in women than in men.

The mood state affects how the subjects describe their personality. Therefore, most previous studies on mood disorders assessed the personality in euthymic period. However, in order to be used as an aid for differentiating unipolar and bipolar depression in a real world clinical setting, it is required to evaluate TCI scores during depressed period. It has been reported that depressed individuals accurately portray their vulnerability to stress, their joylessness, and their lack of motivation, and that depression-caused changes in the assessed personality trait may reflect their current condition of the individual (Costa et al., 2005). Previous studies reported that severity of depression positively correlates with HA and negatively with SD scores (Farmer et al., 2003; Hansenne et al., 1999; Naito et al., 2000; Richter et al., 2000; Spittlehouse et al., 2010). In our study, however, correlation coefficients of HA and SD scores with HDRS scores did not reach statistical significance except for SD in male UP patients. This discrepancy might be due in part to the fact that we did not include patients with a HDRS score of 7 or less.

The fact that TCI scores are influenced by the severity of depression 
complicates the interpretation of the findings. However, the prediction model of BPII for female depressed patients in the present study is unlikely to be greatly biased by the severity of depression for several reasons. First, the mean HDRS scores were similar in BPII and UP patients. Secondly, HA and SD, which are previously reported to be influenced by depression severity, were not included in the prediction model for females. Thirdly, the correlation coefficients relating HDRS scores to each TCI score did not significantly differ between female patients with BPII and UP.

The present study is the first to use personality profiles to create a logistic regression model to predict BPII in depressed patients. Previously, Perlis, et al. (Perlis et al., 2006) made a logistic regression prediction model accurately distinguishing BP and UP by including age at onset, number of previous depressive episodes, family history, Montgomery Åsberg Depression Rating Scale (MADRS) scores, and Hamilton Anxiety Scale scores. Their model predicted bipolarity in depressed patients with a sensitivity of $69.0 \%$ and a specificity of $94.9 \%$, with the total area under the ROC curve of 0.914 . Combining their model with the present one may result in a more accurate prediction model with a wide clinical application. 
A major strength of this study was that patients with BPII and UP were both in depressed state with similar severity of depressive symptoms. To our knowledge, this study is the first to compare the TCI score profiles in BPII and UP patients during depressed states. Knowing the differences in TCI profiles in their depressed states could help clinicians to predict bipolarity in depressed patients.

There are several limitations to this study. First, the crosssectional design did not allow any definitive conclusions as to whether the TCI score profiles of the BPII and UP patients were premorbid or the results of illness onset. Whether the TCI profiles observed here can be generalized to recovered patients needs further investigation. Some UP subjects in this study may go on to experience a manic/hypomanic episode and be rediagnosed as BP, and thus follow-ups are necessary for accurate diagnosis. Secondly, the subjects were recruited through methods such as advertisements and notices, and therefore sampling biases may exist. Thirdly, bipolar patients in our study were limited to BPII. Larger studies are needed to compare the TCI scores between different subtypes of BP or UP. Fourthly, as the BPII and UP patients were limited to those receiving outpatient treatments, our subjects might have been overrepresented by 
milder forms of illness.

In conclusion, we assessed personality profiles in patients with BPII and UP

during depressed period and confirmed that both UP and BPII patients have

characteristic personality profiles in common: higher HA, lower SD, and lower C scores assessed with TCI when compared to controls. However, BPII and UP patients differ in some personality profiles, i.e., higher NS and ST in BPII than in UP patients particularly in female patients. Logistic regression analyses showed that BPII and UP could be predicted based on NS and ST scores in female patients. On the other hand, TCI scores were not very helpful for predicting BPII and UP in male patients. Our findings suggest that assessment of personality profiles using TCI in depressed female patients may serve as a useful tool to conveniently differentiate UP and BPII.

\section{Role of funding source}

Funding for this study was provided by the Intramural Research Grant for Neurological and Psychiatric Disorders of NCNP, Health and Labour Sciences Research Grants (Comprehensive Research on Disability, Health, and Welfare), JST, CREST, and 
"Understanding of molecular and environmental bases for brain health" carried out under the Strategic Research Program for Brain Sciences by the Ministry of Education, Culture, Sports, Science and Technology of Japan (H.K.). They had no further role in study design; in the collection, analysis, and interpretation of data; in the writing of the report; and in the decision to submit the paper for publication.

\section{Conflict of interest}

The authors declare no conflicts of interest.

\section{Acknowledgement}

The authors would like to thank the participants for taking part in the study.

\section{References}

Akiskal, H.S., Mendlowicz, M.V., Jean-Louis, G., Rapaport, M.H., Kelsoe, J.R., Gillin, J.C., Smith, T.L., 2005. TEMPS-A: validation of a short version of a self-rated instrument designed to measure variations in temperament. Journal of Affective 
Disorders 85, 45-52.

Akiskal, H.S., Kilzieh, N., Maser, J.D., Clayton, P.J., Schettler, P.J., Traci Shea, M.,

Endicott, J., Scheftner, W., Hirschfeld, R.M., Keller, M.B., 2006. The distinct

temperament profiles of bipolar I, bipolar II and unipolar patients. Journal of Affective

Disorders 92, 19-33.

American Psychiatric Association, 1994. DSM-IV: Diagnostic and Statistical Manual of

Mental Disorders, 4th Edition. Washington D.C.

Celikel, F.C., Kose, S., Cumurcu, B.E., Erkorkmaz, U., Sayar, K., Borckardt, J.J.,

Cloninger, C.R., 2009. Cloninger's temperament and character dimensions of

personality in patients with major depressive disorder. Comprehensive Psychiatry 50,

$556-561$.

Cloninger, C.R., Svrakic, D.M., Przybeck, T.R., 1993. A psychobiological model of temperament and character. Archives of General Psychiatry 50, 975-990.

Costa Jr., P.T., Bagby, R.M., Herbst, J.H., McCrae, R.R., 2005. Personality selfreports

are concurrently reliable and valid during acute depressive episodes. Journal of

Affective Disorders 89, 45-55. 
de Winter, R.F., Wolterbeek, R., Spinhoven, P., Zitman, F.G., Goekoop, J.G., 2007.

Character and temperament in major depressive disorder and a highly anxious-retarded subtype derived from melancholia. Comprehensive Psychiatry 48, 426-435.

Engstrom, C., Brandstrom, S., Sigvardsson, S., Cloninger, R., Nylander, P.O., 2004.

Bipolar disorder: I. Temperament and character. Journal of Affective Disorders 82,

$131-134$.

Evans, L., Akiskal, H.S., Keck Jr., P.E.,McElroy, S.L., Sadovnick, A.D., Remick, R.A.,

Kelsoe, J.R., 2005. Familiality of temperament in bipolar disorder: support for a genetic spectrum. Journal of Affective Disorders 85, 153-168.

Farmer, A., Mahmood, A., Redman, K., Harris, T., Sadler, S., McGuffin, P., 2003. A sib-pair study of the Temperament and Character Inventory scales in major depression. Archives of General Psychiatry 60, 490-496.

Forty, L., Smith, D., Jones, L., Jones, I., Caesar, S., Cooper, C., Fraser, C., ordon-Smith, K., Hyde, S., Farmer, A., McGuffin, P., Craddock, N., 2008. Clinical differences between bipolar and unipolar depression. The British Journal of Psychiatry $192,388-389$. 
Gutierrez-Zotes, J.A., Bayon, C., Montserrat, C., Valero, J., Labad, A., Cloninger, C.R., Fernandez-Aranda, F., 2004. Temperament and Character Inventory Revised (TCI-R).

Standardization and normative data in a general population sample. Actas Españolas de Psiquiatría 32, 8-15.

Hamilton, M., 1967. Development of a rating scale for primary depressive illness. The British Journal of Social and Clinical Psychology 6, 278-296.

Hansenne, M., Reggers, J., Pinto, E., Kjiri, K., Ajamier, A., Ansseau, M., 1999.

Temperament and character inventory (TCI) and depression. Journal of Psychiatric

Research 33, 31-36.

Hansenne, M., Delhez, M., Cloninger, C.R., 2005. Psychometric properties of the temperament and character inventory-revised (TCI-R) in a Belgian sample. Journal of Personality Assessment 85, 40-49.

Hirano, S., Sato, T., Narita, T., Kusunoki, K., Ozaki, N., Kimura, S., Takahashi, T.,

Sakado, K., Uehara, T., 2002. Evaluating the state dependency of the Temperament and

Character Inventory dimensions in patients with major depression: a methodological contribution. Journal of Affective Disorders 69, 31-38. 
Hirschfeld, R.M., Lewis, L., Vornik, L.A., 2003. Perceptions and impact of bipolar disorder: how far have we really come? Results of the national depressive and manic-depressive association 2000 survey of individuals with bipolar disorder. The Journal of Clinical Psychiatry 64, 161-174.

Kijima, N., Saito, R., Takeuchi, M., Yoshino, A., Ono, Y., Kato,M., Kitamura, T., 1996. Cloninger's seven-factormodel of temperament and character and Japanese version of Temperament and Character Inventory (TCI) Article in Japanese Archives of Psychiatric Diagnostics and Clinical Evaluation 7, 379-399.

Kijima, N., Tanaka, E., Suzuki, N., Higuchi, H., Kitamura, T., 2000. Reliability and validity of the Japanese version of the Temperament and Character Inventory.

Psychological Reports 86, 1050-1058.

Kimura, S., Sato, T., Takahashi, T., Narita, T., Hirano, S., Goto, M., 2000. Typus melancholicus and the Temperament and Character Inventory personality dimensions in patients with major depression. Psychiatry and Clinical Neurosciences 54, 181-189. Loftus, S.T., Garno, J.L., Jaeger, J., Malhotra, A.K., 2008. Temperament and character dimensions in bipolar I disorder: a comparison to healthy controls. Journal of 
Psychiatric Research 42, 1131-1136.

Lovdahl, H., Boen, E., Falkum, E., Hynnekleiv, T., Malt, U.F., 2010. Temperament and character in patients with bipolar II disorder and recurrent brief depression.

Comprehensive Psychiatry 51, 607-617.

Marijnissen, G., Tuinier, S., Sijben, A.E., Verhoeven, W.M., 2002. The temperament and character inventory in major depression. Journal of Affective Disorders 70, 219-223.

Matsudaira, T., Kitamura, T., 2006. Personality traits as risk factors of depression and anxiety among Japanese students. Journal of Clinical Psychology 62, 97-109.

Mendlowicz, M.V., Akiskal, H.S., Kelsoe, J.R., Rapaport, M.H., Jean-Louis, G., Gillin, J.C., 2005. Temperament in the clinical differentiation of depressed bipolar and unipolar major depressive patients. Journal of Affective Disorders 84, 219-223.

Mitchell, P.B.,Wilhelm, K., Parker, G., Austin, M.P., Rutgers, P., Malhi, G.S., 2001. The clinical features of bipolar depression: a comparison with matched major depressive disorder patients. The Journal of Clinical Psychiatry 62, 212-216 quiz 217. Nagelkerke, N., 1991. A note on a general definition of the coefficient of determination. Biometrika 2. 
Naito,M., Kijima, N., Kitamura, T., 2000. Temperament and Character Inventory (TCI)

as predictors of depression among Japanese college students. Journal of Clinical

Psychology 56, 1579-1585.

Nowakowska, C., Strong, C.M., Santosa, C.M., Wang, P.W., Ketter, T.A., 2005.

Temperamental commonalities and differences in euthymicmood disorder patients,

creative controls, and healthy controls. Journal of Affective Disorders 85, 207-215.

Otsubo, T., Tanaka, K., Koda, R., Shinoda, J., Sano, N., Tanaka, S., Aoyama, H.,

Mimura, M., Kamijima, K., 2005. Reliability and validity of Japanese version of the

Mini-International Neuropsychiatric Interview. Psychiatry and Clinical Neurosciences

$59,517-526$.

Pelissolo, A., Lepine, J.P., 2000. Normative data and factor structure of the

Temperament and Character Inventory (TCI) in the French version. Psychiatry Research

94, 67-76.

Perlis, R.H., Brown, E., Baker, R.W., Nierenberg, A.A., 2006. Clinical features of

bipolar depression versus major depressive disorder in large multicenter trials. The

American Journal of Psychiatry 163, 225-231. 
Richter, J., Eisemann, M., Richter, G., 2000. Temperament and character during the course of unipolar depression among inpatients. European Archives of Psychiatry and Clinical Neuroscience 250, 40-47.

Sheehan, D.V., Lecrubier, Y., Sheehan, K.H., Amorim, P., Janavs, J., Weiller, E., Hergueta, T., Baker, R., Dunbar, G.C., 1998. The Mini-International Neuropsychiatric Interview (M.I.N.I.): the development and validation of a structured diagnostic psychiatric interview for DSM-IV and ICD-10. The Journal of Clinical Psychiatry 59 (Suppl. 20), 22-33 quiz 34-57.

Smith, D.J., Duffy, L., Stewart, M.E., Muir, W.J., Blackwood, D.H., 2005. High harm avoidance and low self-directedness in euthymic young adults with recurrent, early-onset depression. Journal of Affective Disorders 87, 83-89.

Spittlehouse, J.K., Pearson, J.F., Luty, S.E.,Mulder, R.T., Carter, J.D.,McKenzie, J.M., Joyce, P.R., 2010. Measures of temperament and character are differentially impacted on by depression severity. Journal of Affective Disorders 126, 140-146.

Tabuse, H., Kalali, A., Azuma, H., Ozaki, N., Iwata, N., Naitoh, H., Higuchi, T., Kanba, S., Shioe, K., Akechi, T., Furukawa, T.A., 2007. The new GRID Hamilton Rating Scale 
for Depression demonstrates excellent inter-rater reliability for inexperienced and experienced raters before and after training. Psychiatry Research 153, 61-67. 